Meta

Journal des traducteurs

Translators' Journal

\title{
L'interjection dans la BD : réflexions sur sa traduction
}

\section{Ascension Sierra Soriano}

Volume 44, numéro 4, décembre 1999

URI : https://id.erudit.org/iderudit/004143ar

DOI : https://doi.org/10.7202/004143ar

Aller au sommaire du numéro

Éditeur(s)

Les Presses de l'Université de Montréal

ISSN

0026-0452 (imprimé)

1492-1421 (numérique)

Découvrir la revue

Citer cet article

Sierra Soriano, A. (1999). L'interjection dans la BD : réflexions sur sa traduction. Meta, 44(4), 582-603. https://doi.org/10.7202/004143ar
Résumé de l'article

Notre travail porte sur les difficultés de traduction de l'interjection dans la bande dessinée. Après en avoir fait l'inventaire à partir de notre corpus, nous en analysons les causes et nous proposons au fur et à mesure des solutions pour améliorer la traduction. 


\title{
L'interjection dans la BD: réflexions sur sa traduction
}

\author{
ASCENSION SIERRA SORIANO \\ Université d'Alicante, Alicante, Espagne
}

\section{RÉSUMÉ}

Notre travail porte sur les difficultés de traduction de l'interjection dans la bande dessinée. Après en avoir fait l'inventaire à partir de notre corpus, nous en analysons les causes et nous proposons au fur et à mesure des solutions pour améliorer la traduction.

\begin{abstract}
In our paper we study the difficulties of translation of the interjection in the comics. We make a classification of them, we analyse the causes and we propose as one goes along some solutions to ameliorate the translation.
\end{abstract}

\section{INTRODUCTION}

Pour les lexicographes du dictionnaire Le Petit Robert, l'interjection est «un mot invariable pouvant être employé isolément pour traduire une attitude affective du sujet parlant». Par contre, le Diccionario de la Real Academia Española indique qu'il s'agit de «una voz que expresa alguna impresión súbita, como asombro, dolor, molestia, amor, etc.» et le Diccionario Enciclopédico Larousse ajoute que:

aunque el RAE, en su gramática, incluye la interjección entre las partes de la oración, la lingüistica moderna no la considera parte (categoría de contenido léxico) ni siquiera partícula (entidad de valor relacionante). Las palabras llamadas interjección son como un rudimento de expresión exclamativo: elemento de exclamación, carente de contenido léxico.

La divergence de ces trois définitions (mot, voz, elemento de exclamación) met en évidence le problème posé par l'interjection, du point du vue linguistique. Celle-ci représente l'un des éléments du langage qui a suscité le plus grand nombre d'opinions contradictoires. La difficulté éprouvée par les linguistes soucieux de l'analyser et d'en élaborer un classement justifie d'emblée l'intérêt qu'on puisse lui porter. Mais si les questions qu'elle soulève dans une langue ont en outre, comme c'est le cas, des répercussions en traduction, au cours de la reformulation dans une autre langue, l'intérêt s'accroît considérablement. Nous envisageons dans ce travail une approche des difficultés de traduction de l'interjection.

\section{LES INTERJECTIONS DANS LA BD}

\subsection{Le concept d'interjection}

L'objectif de ce travail n'étant pas de délimiter les contours du concept «interjection », nous n'en ferons pas une analyse détaillée, mais il convient tout de même de 
cerner la forme et le sens de ce qui constitue notre objet d'étude afin de mieux comprendre, par la suite, les problèmes causés par sa traduction.

L'analyse des conclusions tirées par les linguistes qui se sont intéressés à l'interjection (cf. bibliographie) nous démontre que, malgré leurs divergences, ils ratifient la nature de l'interjection qui est un signe linguistique. Son signifiant peut être soit une forme lexicalisée et expressive, soit une forme lexicale privée de son sens original, soit une combinaison de consonnes et/ou de voyelles qui symbolise un son à l'aide d'une graphie bizarre. Ce mot n'a pas de valeur conceptuelle, mais il signale un ensemble de situations émotionnelles.

Pour ce qui est des fonctions de l'interjection, elles sont au nombre de trois. L'interjection peut être centrée sur le locuteur: dans ce cas, elle est expressive. L'interjection constitue le cas-limite d'expressivité et représente une «marque de subjectivité».

L'interjection peut être centrée sur l'interlocuteur: dans ce cas, elle est appellative - impérative ou interrogative.

Finalement, elle peut être aussi représentative (d'un bruit, d'un cri, d'un son). Cette dernière fonction du langage s'applique par excellence à l'onomatopée, qui, néanmoins, n'est pas toujours inclue dans le même chapitre que l'interjection (cf. par exemple Vigara [1990]; Cascón [1995]). Dans notre étude, nous avons volontairement choisi un corpus composé aussi bien d'interjections que d'onomatopées, puisque nous pensons que ces dernières sont une partie de l'ensemble des interjections.

Notre corpus a été élaboré à partir de six bandes dessinées (trois des aventures d'Astérix et trois de celles de Tintin) et notre analyse se réduit donc à la traduction de l'interjection dans la bande dessinée ${ }^{1}$. Une étude de la traduction de l'interjection en général dépasse largement le format d'un simple article et constituera le sujet d'une monographie.

\subsection{Le langage de la $\mathrm{BD}$}

La BD est, selon la terminologie de Renard (1978:10), un récit dessiné et imprimé ou « une littérature graphique» (Francis Lacassin) ou encore « une figuration narrative» (Pierre Carperie). L'enchevêtrement de l'écriture (du texte), du dessin (de l'iconisme) et du bruitage (de l'onomatopée visuelle ou phonique) définit le langage typique de la BD. Le texte et l'image coexistent donc et doivent s'entremêler pour rendre «l'illusion de la vie» en imageant le mouvement, les sons, la psychologie des personnages. Il est vrai, en effet, que les signes iconiques permettent la description d'une façon précise et réaliste, mais c'est le langage verbal qui est le plus adéquat pour transcrire les émotions et les pensées des personnages et qui exprime mieux les sentiments que l'image.

L'une des caractéristiques du langage des ballons de la BD, qui prend de plus en plus d'importance au détriment du texte hors de l'image qui devient uniquement illustratif, est effectivement l'emploi systématique du style direct. De plus, dans la BD, on ne peut que constater la primauté de la fonction de "pseudo-communication de personne à personne» dont parle Fresnault-Deruelle (1972b:15): les appellatifs, les injures, le vocabulaire de l'action, de l'imprécation, l'interrogation constituent l'ample majorité du texte, les relations interpersonnelles se révélant très importantes ou même le plus important. 
Caractère appellatif donc du texte de la BD! Si l'on y ajoute le caractère expressif que nous avons abordé auparavant et le caractère représentatif fourni par les onomatopées, ne retrouvons-nous pas les trois fonctions caractéristiques de l'interjection? L'apparition de l'interjection dans cette langue parlée utilisée dans la BD va donc de soi; son emploi ne se produit pas par hasard, mais de façon tout à fait consciente et voulue. Les auteurs recherchent l'interjection, d'une part, parce qu'elle est véritablement très employée dans la langue parlée réelle, parce qu'elle est apte à rendre les affects et les idées spontanées des individus, et, d'autre part, parce qu'elle participe à tous les égards de la langue parlée littéraire qui caractérise la $\mathrm{BD}$ : celle qui transmet au lecteur l'impression de vie, de dynamisme et d'action.

\subsection{L'importance des interjections dans le langage de la $\mathrm{BD}$}

L'importance des interjections dans les BD du point de vue quantitatif s'explique et n'a plus rien d'étonnant après cette analyse du langage des BD.

Les données numériques sont très significatives: notre corpus est constitué de 166 interjections non onomatopéiques (96 dans Tintin et 70 dans Astérix) et de 178 onomatopées (63 dans Tintin et 115 dans Astérix), soit un total de 344 interjections dans six albums.

Ce nombre d'interjections s'accroît si l'on comptabilise également les répétitions de la même interjection (par exemple, ah! figure onze fois dans Astérix et dix-neuf fois dans Tintin) ou les différentes graphies de la même interjection (par exemple, ah! - aaah! , oh! - oooh!).

Finalement, une autre donnée numérique souligne l'importance de l'interjection dans la BD: dans Astérix le Gaulois, album qui comporte 48 pages, figurent 56 vignettes dont le texte est composé exclusivement d'une interjection, soit au moins une vignette par page qui ne contienne pour tout message verbal qu'une interjection.

Pour obtenir une bonne traduction de la $\mathrm{BD}$, il est évident que, d'une part, outre l'obligation de la part du traducteur de connaître à fond les différentes valeurs de l'interjection et ses différents emplois dans la langue parlée française, la connaissance exhaustive du code et des techniques d'expression de la BD est nécessaire pour pouvoir rendre à bon escient la même réalité et conserver la fidélité à la $\mathrm{BD}$ d'Hergé ou de Goscinny, malgré la brièveté du texte, caractère qui ne peut absolument pas être changé dans la traduction. D'autre part, il faut bien sûr une excellente connaissance de l'espagnol familier et de l'emploi de l'interjection qui en fait partie.

Nous pouvons prétendre, sans exagérer il nous semble, que traduire une BD consiste surtout à traduire des interjections ou, tout au moins, que la traduction des interjections constitue un énorme travail et une partie significative dans le processus de traduction d'une BD.

\section{TRADUCTION DE L'INTERJECTION DANS LA BD}

Le but de ce travail est par conséquent d'analyser les différentes traductions de l'interjection en comparant les textes originaux en français et les textes traduits en espagnol et de mettre en relief les difficultés rencontrées pour les traduire. 


\subsection{Classement des interjections et leur traduction dans la BD}

Les difficultés pour définir l'interjection en tant que signe linguistique, dues au manque de valeur conceptuelle, à la complexité de sa forme, à son «détachement»syntaxique, à sa valeur pragmatique, à ses rapports étroits avec le cotexte et le contexte, etc., débouchent inévitablement sur les problèmes de classement de l'interjection. C'est pourquoi nous nous trouvons face à toutes sortes de classements qui envisagent l'interjection d'un point de vue soit sémantique, soit morphologique, soit syntaxique (cf. bibliographie).

Notre classement des interjections, particulier puisqu'il est réalisé en vue d'une traduction, prend en considération non seulement des interjections françaises, mais aussi les possibilités de regroupement des équivalents de traduction obtenus en espagnol. C'est pourquoi nous refaçonnons les différents modèles de classements en introduisant toutes les divisions que nous jugeons nécessaires pour établir un parallélisme entre les deux langues. Le classement est morphologique au départ mais, pour chaque sous-partie, nous envisageons un classement sémantique, sémiotique ou d'ordre pragmatique selon les emplois de l'interjection dans la communication. Il serait dommage de se priver de toutes ces données puisque le résultat obtenu (les conclusions sur les équivalents de traduction) y gagnera inéluctablement en clarté...

\subsubsection{Les bruits}

Les sons d'objet, qui imitent les bruits de la nature, ne sont pas des données sensorielles qui se sonorisent spontanément; on a affaire en fait à une création linguistique qui imite la réalité en se soumettant aux règles de la langue commune. C'est pourquoi, pour comprendre une onomatopée dans une langue, il faut connaître cette langue, car les onomatopées, même si elles reproduisent le même bruit, diffèrent sensiblement d'une langue à une autre, d'une convention linguistique à une autre. Comme disait Sauvageot (1964:180), «les images vocales n'ont de signification que dans la mesure où on est initié». L'analyse de notre corpus démontre que le traducteur connaît cette condition; il cherche donc à traduire les sons artificiels français par un équivalent dont la forme correspond au système morphologique espagnol:

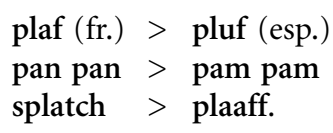

Cependant, l'influence des BD américaines se fait nettement sentir pour ce genre d'onomatopées; surtout dans la reproduction de bagarres pendant lesquelles les coups sont accompagnés de bruits secs et forts, de heurts violents, l'assimilation des reproductions onomatopéiques anglaises dans les traductions de BD est pratiquement totale. Les onomatopées bing bang boom clong paf bif splok tchoc... s'intègrent de façon si absolue au dessin imageant une bataille qu'elles sont devenues des conventions du code pictural de la $\mathrm{BD}$ et se transforment ainsi en «sons visuels» qui s'appliquent automatiquement, tels quels, à une situation concrète aussi bien en français qu'en espagnol. C'est la «fonction imageante» du texte dont parle FresnaultDeruelle (1972c:43).

On trouve néanmoins quelquefois une adaptation graphique qui surprend, car dans la même vignette, il est facile de voir côte à côte bum, forme tout à fait espa- 
gnole et tchrraaaac dont la composition initiale de consonnes révèle une origine étrangère.

Cette soumission au modèle original américain réapparaît:

- au moment de l'adaptation graphique en espagnol: à ce moment-là se produisent des erreurs causées par l'emploi de consonnes et de morphèmes peu espagnols:

dzing > cling (alors que le " $\mathrm{g}$ » se perd en espagnol dans les terminaisons qui proviennent du suffixe «-ing» anglais);

- ou dans le choix de ne pas faire d'adaptation graphique, dans le cas des emprunts: pourtant, une simple adaptation graphique suffirait pour intégrer l'élément au système espagnol:

choc au lieu de tchoc,

drin au lieu de dring (sonnette),

tin tin tin au lieu de ting ting ting (cogner le verre avec un couvert),

zzz au lieu de wzzz (bruit d'un projectile).

Parfois, le traducteur maintient le terme étranger dans sa traduction; il emprunte, cette fois-ci, le mot français alors qu'il existe des équivalents espagnols:

- rreuh (anglicisme du français) > bruuutbruut (mot à forme française) qui devrait être brum brum (accélération d'une voiture en espagnol);

- toot (forme anglaise empruntée en français) > tuuut (forme française empruntée en espagnol) alors qu'on a piit piit en espagnol (klaxon);

- ding ding dong (fr.) > ding ding dong (esp.) au lieu de din don (pour une cloche) ou dilin dilin dilin (pour une clochette).

\subsubsection{Les «voix» des animaux}

Les sons qui reproduisent les cris des animaux ont généralement une forme autochtone que le traducteur utilise:

cocorico $>$ quiquiriqui ou kikiriki
ouah $>$ guau
meuh $>$ muh.

\subsubsection{Les cris inarticulés}

\subsubsection{Des animaux}

Par contre, les cris inarticulés d'animaux ne sont pas traduits. Par exemple, quand le chien de Tintin poursuit un chat, celui-ci se rebiffe et, montrant les dents, crie de la manière suivante: fffh- gu- schh. Ce cri est conservé tel quel en espagnol. Cette forme symbolise l'air qui sort des poumons du chat furieux et n'importe quel locuteur sait fort bien la difficulté pour un homme d'imiter les cris animaux. Le traducteur compte donc sur l'agilité mentale et la connaissance des codes implicites de la BD de la part du lecteur pour que celui-ci comprenne cette onomatopée sans besoin de la traduire.

\subsubsection{Des hommes}

En revanche, la prise de position du traducteur semble un peu hésitante puisque, dans la même situation de sons inarticulés mais proférés, cette fois-ci, par un humain, il décide de réorganiser des combinaisons consonantiques, ce qui ne facilite en 
aucune façon la prononciation et n'apporte donc aucune aide substantielle au lecteur. On est en droit de se demander par exemple la raison des équivalents GNGNGN au lieu de RGNRGNRGN ou GMGMGMMY pour traduire MMGNOMMGNOMGNON par opposition à NNNG, qui ne change pas dans le texte traduit.

Il est vrai cependant que la tendance est celle d'adapter l'onomatopée :

par exemple, TSK TSK TSK > SST SST

en introduisant souvent un son vocalique qui permet de mieux prononcer en espagnol:

hmgmpffpff $>$ jijiji;

hmm > humm (par contre, la représentation hmhmm reste la même!).

Il faut toutefois reconnaître le mérite du traducteur qui, en tant que lecteur de $\mathrm{BD}$, connaît les conventions suivantes: plusieurs zzzz ou un $\mathrm{Z}$ majuscule indiquent toujours le sommeil, et une suite de lettres telle que MRKRPWZKRR remplace les gros mots, les mots tabous, qu'emploie un personnage qui se met en colère (par exemple, le capitaine se fâche parce que la bouteille est vide (TT:26). Mais aucun des spécialistes qui ont analysé la BD (cf. Fresnault-Deruelle, Benayoun, Gubern, etc.) ne fait référence à la conventionnalité du graphème $\mathrm{R}$ pour transcrire le ronflement et pourtant, dans notre corpus, l'onomatopée RRR n'est pas traduite.

\subsubsection{Les sons articulés humains}

Parmi les sons humains, les sons articulés constituent évidemment la grande majorité. Nous les présentons en deux parties, les interjections propres d'une part (c'est-àdire mot qui n'existe que dans cet emploi) et les interjections impropres d'autre part (mot qui emprunte sa forme à un autre signe linguistique).

\subsubsection{Interjections propres}

Les interjections propres sont, selon leur fonction, représentatives (onomatopée qui imite un bruit naturel), appellatives (qui s'orientent vers l'interlocuteur) et expressives (qui traduisent un sentiment, ou une attitude manifestés par le locuteur).

\subsection{Onomatopées}

Les onomatopées de notre corpus symbolisent:

- des cris (par exemple, de douleur: aïe, ouille);

- des bruits phonatoires:

- le nez qui renifle (snif > snif, ce terme étant un anglicisme utilisé dans les deux langues),

- le bruit de la gorge quand nous buvons (glou glou > glu glu);

- ou des phénomènes physiques:

- l'éternuement (atchoum > atchiss),

- le hoquet (hic > hip),

- le soupir (ouf $>$ uf),

- le rire (hahaha $>$ jajaja hihihi $>$ jijiji hohoho $>$ jojojo),

- les pleurs (bouhouhouhou $>$ bubububu ou buaaa — forme qui nous semble nettement plus espagnole), etc. 


\subsection{Interjections appellatives}

Quant aux interjections appellatives, nous trouvons par exemple:

- pour les interjections adressées à un interlocuteur humain:

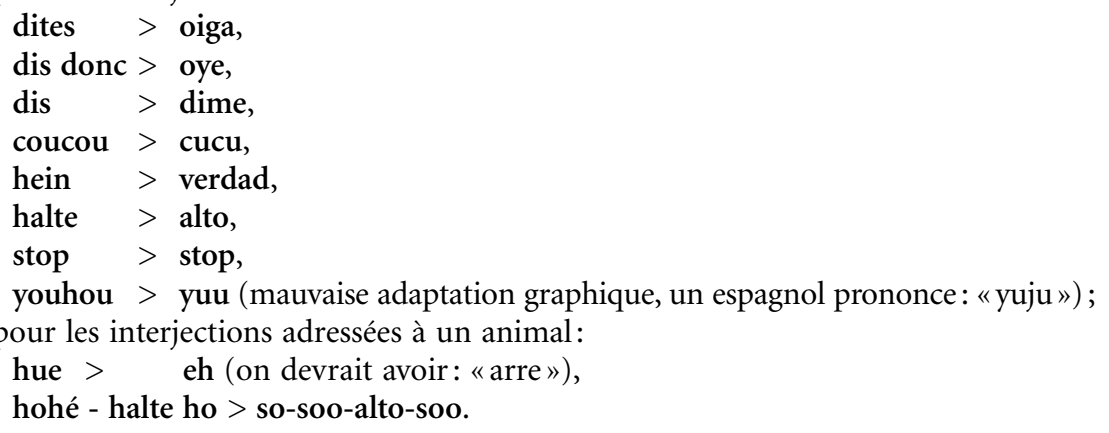

\subsection{Interjections expressives}

Pour ce qui est des interjections qui transmettent les affects de la personne, nous pouvons citer celles qui indiquent:

- un échec:

zut alors $>$ mecachis,

zut $>$ ay, buf (mauvaise traduction);

- la surprise:

ho $>$ oh - oh,

ah $>$ ah (ou pas de traduction),

ça alors > eso sí que es... (on aurait pu avoir: «vaya, anda»);

- la négation:

taratata > qué va! (on aurait pu avoir l'interjection: «nananana»);

- la peur:

kaï kaï > ay ay,

hui $>$ ayy, aie $>$ ay Dios mío, Dios mío;

- le plaisir de manger: miam miam > ñam ñam;

- le désir de s'affirmer: na $>$ toma;

- la demande de silence: chut $>$ silencio (qui correspondrait plutôt à : silence!), calla (qui correspondrait à : tais-toi!), chist, psit;

- l'hésitation: euh - euh > pues, ejem;

- la réaction devant une situation différente: oh là là $>$ oh là là (erreur sans aucun doute, car un espagnol ne comprend pas cette interjection), aïe aïe aïe > ayy;

- la satisfaction: chic > jiji, chist, glub (dans les trois cas, erreur due à la mauvaise interprétation de la situation), hale (interférence linguistique: français : «allez»), estupendo;

- la joie: hourrah > hurra;

- la lamentation: hélas > por desgracia, hay, desgraciadamente, lo siento; 
- le constat ou le soupçon:

tiens $>$ vaya, vaya vaya,

voyons > veamos;

- le désir d'enlever de l'importance à quelque chose: bah > bah;

- le désir de transgresser une norme, blasphémer: saperlipopette $>$ carambita, parbleu > ah! sí!, sapristi > vaya, hem, caramba, mecachis, canastos;

- le début d'une action du locuteur: hop > hop (qui devrait être: «jop»), sans traduction, allons-y $>$ andando, pues toma,

- le fait qu'on admet quelque chose: ma foi $>$ anda (mauvaise traduction par une interjection qui dénote la surprise du locuteur).

D'autres sont de simples charnières dans la conversation et fonctionnent comme des tics de langage dans la langue française essentiellement. C'est pourquoi, très souvent, le traducteur les omet en espagnol:

eh bien > pues, bueno;

ben $>$ es que, eh ben.

Toutes ces interjections propres, qu'elles soient de nature onomatopéique ou non, ont presque toutes un équivalent de traduction espagnol utilisé par le traducteur. Mais force est de remarquer le grand nombre de traductions erronées, à notre avis, enregistrées pour ce type d'interjections.

Les raisons en sont principalement une mauvaise compréhension de la situation de communication, due sans doute à l'inexpérience du traducteur en tant que lecteur de $\mathrm{BD}$, ou à une connaissance incomplète d'une des deux langues mises en parallèle, ce qui provoque des interférences, des faux sens, de mauvaises adaptations graphiques, et les différentes valeurs qu'acquièrent certaines interjections (en perdant leur origine onomatopéique, selon le cotexte ou leurs connotations extralinguistiques). Mais il en est d'autres. Ainsi, une difficulté surgit quand il n'existe pas d'interjection équivalente (pour hélas, heu par exemple) ou au contraire quand il existe plusieurs équivalents parmi lesquels il convient de choisir le plus fidèle à la forme du signe original ou au sens (par exemple, chut - chiss et non pas silencio). Nous nous contentons de nommer à ce propos quelles sont les formes de traduction proposées et quelques difficultés rencontrées pour traduire l'interjection, car nous ferons ensuite l'étude de ces dernières de façon plus systématique.

\subsubsection{Interjections impropres}

Quant aux interjections impropres, elles sont numériquement beaucoup moins nombreuses et nous proposons pour analyser leur traduction de les diviser en deux groupes suivant leur orientation locutoire ou interlocutoire.

\subsection{Orientation locutoire}

Les éléments du premier groupe réunissent les jurons et l'expression des sentiments du locuteur. 


\subsection{Jurons}

Les jurons sont des jurements qui transgressent linguistiquement un code moral ou religieux. «Le locuteur prend l'allocutoire à témoin pour jurer contre quelque chose tenu pour sacré ou tabou par la collectivité en nommant précisément les mots sacrés ou tabous» (Olivier 1994:227). Il s'agit d'une décharge émotive du locuteur.

Le juron est, à l'origine, un jurement d'outrage qui «procède du besoin de violer l'interdiction biblique de prononcer le nom de Dieu», une blasphémie (Benveniste 1974, chap. 14 «La blasphémie et l'euphémie»: 254). Dans notre corpus figurent par exemple:

par tous les Dieux, mes Dieux, grands Dieux, Dieu, mon Dieu.

Toutes ces formes ont un équivalent espagnol qui conserve le terme «Dieu»:

por todos los Dioses, Dioses míos, Dios mío, Dios.

Si l'on fait référence à d'autres religions, le traducteur doit respecter ces croyances et leur vocabulaire. Nous trouvons ainsi dans Astérix:

par Jupiter $>$ por Júpiter;
par Thor $>$ por Tor;
par Odin $>$ por Odin;
par Toutatis $>$ por Tutatis.

Mais ce besoin de bafouer le divin, de blasphémer suscite toujours une censure de la communauté linguistique et par conséquent, en même temps, une formulation atténuante, moins outrageante: certains jurons ont donc une structure lexicale euphémique.

L'euphémisme est obtenu de différentes manières (cf. Benveniste 1974) :

- en remplaçant «Dieu» par un autre terme: au nom de Dieu devient au nom du ciel!;

- en remplaçant «Dieu» par une même assonance: par Dieu devient parbleu!;

- en créant une forme composée qui n'a pas de sens en dehors de l'interjection: sacré Dieu devient sapristi ou saperlipopette.

(Dans les deux derniers cas, il s'agit d'interjections propres qui sont déjà apparues sous cette rubrique).

Toutes ces formes euphémiques ont été bien traduites par un équivalent espagnol qui généralement est un mot «innocent», une autre interjection qui varie selon le sentiment ou l'attitude exprimés par le locuteur:

parbleu $>\nmid$ ah $! \nmid s i$ ! (insiste sur l'affirmation);

saperlipopette $>$ ¡ carambita! $¡$ adios! (indique la surprise);

sapristi > vaya, canastos, caramba (surprise), mecachis (lassitude, ennui), hombre (énervement);

ou au contraire, une interjection dans laquelle apparaît le mot «Dios», sans plus :

au nom du ciel $>$ Dios mío, en nombre de Dios. 


\subsection{Expression des sentiments du locuteur}

En ce qui concerne les interjections impropres qui traduisent les sentiments du locuteur, celles de notre corpus peuvent exprimer:

- la surprise:

sans blague $>$ ¡ ésta sí que es buena!,

ma parole $>$ anda, toma,

par exemple > vaya, caramba, asombroso, esto sí que...;

- l'approbation:

parfait! oh! j'ai compris > pas de traduction directe (son sens est rendu par la répétition de «lo comprendo, lo comprendo»),

bon > bueno,

extra, génial, super-dément $>$ extra, génial, super-sensacional;

- l'inquiétude:

malheur! > ¡malo!,

maman!> ¡mamá!.

À ces exemples, il faut ajouter un cas particulier: les interjections formulées par le capitaine Haddock dans les albums de Tintin, qui expriment parfois sa surprise, parfois son indignation, ou son étonnement, etc., mais qui sont toujours composées à partir de «tonnerre », «sabords » et «Brest» et qui se combinent avec «millions » ou «milliards» pour former des expressions:

tonnerre $>$ demontre, caramba, rayos y truenos, diablos, diablo, trueno;

mille tonnerres $>$ recaramba (pas de traduction dans un cas);

diable > caramba ;

tonnerre de tonnerre de Brest $>$ rayos y centellas, truenos y centellas;

mille millions de mille milliards de mille sabords de Tonnerre de Brest $>$ rayos y centellas, mil millones de millones de demonios;

mille sabords $>$ mil rayos, centellas;

Tonnerre de Brest $>$ truenos y centellas, rayos y truenos;

mille milliards de mille sabords de Tonnerre de Brest $>$ mil millones de naufragios mil millones de demonios, rayos y centellas;

mille millions de mille de Tonnerre de Brest $>$ mil millones de sabords relámpagos, caramba con el demonio.

Les traductions employées se composent d'interjections courantes (propres: caramba, demontre, recanastos, ou impropres : diablos, demonios) et d'unités lexicales issues du même champ sémantique: truenos, rayos, centellas, relámpagos. Mais toutes ces formes sont traduites de plusieurs façons au long du récit: cette prolifération d'équivalents pour le même terme et de la part du même traducteur ne semble pas adéquate. Elle supprime, d'une part, l'effet comique produit par la répétition de la même interjection en français et, d'autre part, la catégorie de «tic de langage» dans laquelle figure cette interjection dans le texte et qui caractérise outre mesure la façon de parler du capitaine.

Du point du vue syntaxique, on peut remarquer qu'il y a un transfert de la catégorie grammaticale du mot qui compose l'interjection impropre au cours du passage d'une langue à une autre: un adjectif peut être traduit par un adjectif mais un substantif peut l'être par un adjectif, par une forme verbale ou par une phrase exclamative. 
Le changement sémantique de ces interjections qui traduisent en français les sentiments du locuteur et la spécialisation de leur charge émotive dans des contextes différents provoquent de plus une sérieuse difficulté pour le traducteur: il doit faire preuve d'une créativité plus grande pour rendre la même valeur sémantique. La traduction de ces interjections souvent improvisées, créées par les auteurs pour accrồtre l'illusion de vie chez leurs personnages dans leur récit, oblige le traducteur-lecteur de $\mathrm{BD}$ à inventer lui aussi; il peut et doit donner libre cours à sa fantaisie linguistique en jouant avec les catégories grammaticales, les unités lexicales et les expressions exclamatives. C'est dans ce cas concret que nous trouvons le plus de liberté ou de distance entre les équivalents des deux langues et c'est à ce moment que le traducteur doit faire montre de son savoir-faire linguistique dans la langue d'arrivée.

Cependant, cette plus grande marge de création peut aussi conduire inexorablement à des erreurs de traduction, comme par exemple:

poil au menton, traduit par buena idea.

\subsection{Orientation interlocutoire}

Pour ce qui est des interjections à orientation interlocutoire, elles peuvent être centrées :

- sur un ensemble de personnes inconnues, sur tous ceux qui puissent l'entendre:

* on demande de l'aide:

à moi ! > a mi (qui ne s'utilise pas en espagnol),

au secours > pas d'équivalent (pourtant il existe: ¡auxilio!, ¡socorro!);

* on prévient d'un danger imminent:

alerte, alerte! > ¡alarma, alarma!,

attention > atención (qui dans la langue parlée espagnole ne s'emploie pas; on pourrait avoir: ¡cuidado! ou l'interjection ¡ojo!);

- sur un individu:

* pour le féliciter:

bravo! > ¡bravo!;

* pour l'encourager:

hardi! > ¡ánimo!;

* pour le plaindre:

malheureux! > ¡desgraciado! (la traduction serait plutôt d'après le contexte: infeliz);

* pour l'insulter:

gâcheur! > ¡aguafiestas!,

tête de mule > ¡cabeza de mula!;

- sur un groupe de personnes bien délimité: dans ce cas, nous n'avons affaire qu'à des injures qui, dans notre corpus, sont toutes proférées par le même personnage, c'est-àdire le capitaine Haddock, dans les albums de Tintin. Nous donnerons quelques exemples tirés des Sept boules de cristal: 


\begin{tabular}{|l|l|c|}
\hline $\begin{array}{l}\text { sauvages, gredins, } \\
\text { analphabètes, va-nu-pieds, } \\
\text { ectoplasmes, cornichons }\end{array}$ & $\begin{array}{l}\text { salvajes, analfabetos, } \\
\text { desgraciados, ectoplasmas, } \\
\text { calabacines }\end{array}$ & 43 \\
\hline $\begin{array}{l}\text { tas de sauvages, vampires, } \\
\text { protozoaires, brutes }\end{array}$ & $\begin{array}{l}\text { banda de caníbales, } \\
\text { vampiros, brutos }\end{array}$ & 45 \\
\hline $\begin{array}{l}\text { bande de canaques, pirates, } \\
\text { bachi-bouzouks }\end{array}$ & banda de canacas, piratas & 47 \\
\hline $\begin{array}{l}\text { bandits, chauffards, } \\
\text { saltimbanques, écraseurs, } \\
\text { prutes, nyctalopes, }\end{array}$ & $\begin{array}{l}\text { bandidos, saltimbanquis, } \\
\text { avasalladores, brutos, } \\
\text { nictálopes, parásitos }\end{array}$ & 55 \\
\hline $\begin{array}{l}\text { cornichons, moules à gaufres, } \\
\text { zoulous }\end{array}$ & $\begin{array}{l}\text { calabacines, zopencos, } \\
\text { zulús }\end{array}$ & 55 \\
\hline $\begin{array}{l}\text { analphabètes, crétins } \\
\text { des Alpes }\end{array}$ & analfabetos, cretinos \\
\hline garnements, bandits, iconoclastes Alpes & arrapiezos, bandidos, ah, si os pesco & 59 \\
\hline tête de mule & no seas borrico & 60 \\
\hline
\end{tabular}

De l'étude des interjections impropres, il ressort que celles-ci sont l'objet d'une recherche minutieuse de la part du traducteur, car leurs caractéristiques formelles le poussent à bien comprendre leur emploi dans la situation d'énonciation. Elles ont pratiquement toujours un équivalent de traduction, stéréotypé ou créé de toutes pièces. Ce dernier type d'équivalents, ceux qui sont créés par le traducteur, sont plus précisément destinés à des traductions inadéquates.

\subsubsection{Conclusion}

En synthèse, les méthodes adoptées par le traducteur pour traduire l'interjection dans la $\mathrm{BD}$ sont les suivantes:

- fournir un équivalent de traduction pour les bruits de la nature, bien que l'influence des BD américaines favorise l'apparition d'emprunts à l'anglais (surtout pour reproduire des chocs violents et, généralement, lorsque l'onomatopée devient partie intégrante de l'image);

- utiliser l'équivalent espagnol des différentes "voix» d'animaux, mais conserver la formulation inarticulée de certains cris qui sont exclusivement composés de consonnes;

- adapter les sons inarticulés humains, c'est-à-dire recombiner les consonnes employées en y ajoutant parfois un son vocalique;

- proposer une traduction pour les interjections propres(cependant, il faut remarquer que le plus grand nombre d'erreurs se produit dans ce cas particulier: souvent, l'équivalent ou l'omission de l'équivalent ne rend pas le même effet dans le texte que le terme original);

- trouver ou créer un équivalent de traduction pour les interjections impropres (jurons, expression des sentiments face à une situation déterminée ou envers un interlocuteur); dans ce cas, le traducteur a recours à plusieurs procédés: 
- trouver un équivalent «lexicalisé»,

- créer un équivalent qui s'adapte au système linguistique espagnol et qui possède la même valeur énonciative dans le discours,

- supprimer l'interjection mais rendre le même sens au moyen d'une autre structure syntaxique ou en ajoutant un texte explicatif (ma parole! > jésta sí que es buena!),

- compenser une certaine perte de charge émotive ou un léger changement sémantique par d'autres signes orthographiques ou typographiques.

Donc, le souci et le besoin de traduire l'interjection, qu'elle soit de nature onomatopéique ou pas, apparaissent clairement; pour ce faire, on utilise un équivalent total ou partiel, on adapte une forme étrangère, même si parfois le code pictural de la $\mathrm{BD}$ gagne du terrain aux dépens du code linguistique, l'image l'emportant sur l'interjection (et c'est dans ce cas seulement qu'abondent les anglicismes!).

Ce travail de recherche traductologique, louable et jusqu'à présent peu loué, car peu reconnu dans le domaine de la linguistique comparée, est laborieux et difficile. La preuve en est, nous l'avons souligné partiellement au fur et à mesure de notre analyse, l'existence d'erreurs fréquentes d'adaptation morphologique et graphique, de confusions de sens ou d'emplois surprenants.

C'est pourquoi nous avons voulu relever dans la dernière partie de ce travail les différentes difficultés auxquelles est confronté le traducteur qui traduit l'interjection dans les $\mathrm{BD}$, en donner des exemples concrets, puisés dans notre corpus afin de les classer et de les analyser.

\subsection{Difficultés que pose la traduction des interjections dans la BD}

\subsubsection{La connaissance insuffisante des langues}

Le facteur qui conditionne le plus une traduction est évidemment la connaissance plus ou moins parfaite des deux systèmes linguistiques comparés. Les difficultés rencontrées dans la traduction de l'interjection dans la BD sont d'ordre morphologique (problème des onomatopées, des graphies), d'ordre lexico-sémantique (question des emprunts, des faux-sens...) et d'ordre stylistique.

\subsubsection{Difficultés d'ordre morphologique}

\subsection{L’onomatopée}

L’onomatopée est un phénomène purement conventionnel. La représentation morphologique des cris d'animaux par exemple, qui longtemps a été considérée comme tout à fait motivée, n'est en fait qu'un processus de symbolisation; les interjections onomatopéiques sont des mots phonosymboliques dont il est facile d'augmenter le nombre en introduisant de nouvelles créations imitatives.

Il s'agit d'un signe inventé pour reproduire toutes sortes de bruits et de mouvements, d'une abstraction linguistique qui est, de plus, conventionnelle: c'est-à-dire que pour "savoir ce que veut dire une onomatopée dans une langue donnée, il faut apprendre la langue en question, ce qui revient à dire qu'il faut connaître par suite de quelle convention telle séquence de sons a tel ou tel sens plus ou moins déterminé» (Sauvageot 1964:180). 
Par exemple, un traducteur qui ignore que l'onomatopée tacatán tacatán représente en espagnol le galop du cheval (Beinhauer, 1991:101) peut omettre la traduction comme c'est le cas dans notre corpus $(A N: 13)$. Il commet ainsi une erreur puisque la formulation empruntée au français cotcotcot n'indique pas en espagnol le même bruit et ressemble davantage au cri de la poule! (cf. cooc proposé pour coot...codec $[A N: 13])$. Un autre exemple tiré de notre corpus est celui du son de la trompette: le traducteur le transcrit de la même façon en espagnol qu'en français, tariii taraaa!, alors qu'en espagnol, l'onomatopée conventionnelle est ¡tututuuu! (ATG:33).

D'autre part, dans le cas où l'une des deux langues s'impose chez le traducteur, il est extrêmement facile que se produisent involontairement des glissements de certains éléments de cette langue dans l'autre langue. Tel est le cas des interférences phonétiques:

- Par exemple, quand Idéfix pleure, apparait bububu (traduction de bouhouhou) au lieu de buaaa qui serait plus correct ( $A N: 23)$. Ici s'est glissée l'assonance française en [u] dans l'onomatopée du texte espagnol.

- Ou la traduction de allez! (dans allez, viens! ou allez, on fonce!) par ¡hale! (¡hale, al ataque!). L'auteur considère sans doute que cette forme est onomatopéique mais, en réalité, elle illustre bien le cas d'une interférence du français dans le domaine phonétique: les sons français [ale] ont été transcrits par une graphie soi-disante espagnole (ATG: 6 et 25$)$.

\subsection{La graphie}

Par ailleurs, les systèmes graphiques français et espagnol doivent être connus du traducteur. Cela éviterait par exemple la traduction de tchouf par ¡ chouf! (graphie française «ou») au lieu de ¡ chuf!, ou de tchoc! par le même mot au lieu de ¡ choc!, ou, finalement, la reprise telle quelle de dring, cling, ring! au lieu de ¡drin, clin, rin!

Cela faciliterait par exemple le passage de youhou! à ¡ yuju! et non ¡ yuu! (de la même façon que hihihi devient jijiji par exemple) ou le transfert de hop ! à i jop !, qui est la transcription de l'interjection réellement utilisée en espagnol.

\subsubsection{Difficultés d'ordre lexico-sémantique}

Le deuxième type de difficultés que nous étudions est d'ordre lexico-sémantique. Il fait référence aux emprunts à l'anglais, d'une part, aux interférences qui se produisent dans le transfert d'une langue à l'autre des formes lexicales et, d'autre part, à la connaissance insuffisante de la langue de départ.

\subsection{Les emprunts}

Les deux types de BD analysées, Astérix et Tintin, sont littéralement envahies par des formes lexicales qui révèlent clairement leur origine étrangère. En effet, l'anglais possède une extrême richesse en substantifs et verbes phonosymboliques (cf. Santoyo 1985: 170 ou Gubern 1974:153), par exemple:
to ring;
to smash;
to crunch;
to clap;
to skruntch;
to blow;
to ding;
to crash;
to click;
to crack;
etc.
to knock;
to splash;
to sniff; 
qui sont à la base des onomatopées dans les BD américaines. Celles-ci ont été ensuite exportées et elles sont entrées massivement dans les BD franco-belges, puis dans leur traduction en espagnol (dans le cas qui nous intéresse), sans tenir compte des graphies totalement inusuelles, des combinaisons de graphèmes ou de sons tout au moins bizarres.

Les bédéistes, sur leur lancée, en sont même arrivés à l'extrême de reproduire les bruits par des graphies imprononçables dans un essai de traduire graphiquement tous les sons de la nature. Comme le dit Fresnault-Deruelle (1971:81), «la multiplication de ces mots "barbares" apparaît comme une tentative désespérée de vaincre l'inertie de l'écriture».

Mais l'adoption dans les traductions espagnoles de ces formes telles quelles, ces emprunts à l'anglais par le biais de la langue française soulèvent plusieurs problèmes. En effet, certains anglicismes employés dans la langue française le sont également dans la langue espagnole; dans ce cas, l'équivalence est totale et sans difficultés. Mais il arrive que certains anglicismes français aient des équivalents de traduction en espagnol dont la forme appartient au système morphologique espagnol. Ce phénomène provoque alors la création d'un doublet: un anglicisme qui est utilisé sans transformation dans la langue française mais pas toujours dans la langue espagnole et un signe dont le signifiant suit les règles de formation morphologique de l'espagnol. Par exemple:

$$
\text { dring (angl.) > dring (fr.) > dring ou drin (esp.). }
$$

Nous pouvons même trouver certains cas où se côtoient plusieurs signes de trois provenances différentes (anglais, français, et espagnol):

boom (angl.) > boom ou boum, baoum (fr.) > (esp.) boom, boum, baoum, bum, pum.

On est en droit de se poser des questions sur l'utilité d'un tel procédé et sur les raisons de l'apparition mais aussi de l'acceptation généralisée de cette difficulté que représente en fait l'existence parallèle de plusieurs signes.

De l'analyse de ces onomatopées, il ressort que, très souvent, elles sont traduites quand elles apparaissent dans un texte verbal à l'intérieur d'un ballon, mais qu'elles ne le sont pas quand elles constituent le texte non entouré. Ce texte est la manifestation d'un type de ballon, le ballon-zéro; il est très réduit et traduit soit un cri, soit un bruit. Dans ce cas-là, l'expressivité acoustique de ces termes devient expressivité plastique. L'onomatopée s'intègre à l'image, elle devient partie intégrante du pictogramme, elle se transforme en idéogramme. «Le «bruit» du ballon-zéro se caractérise par son aspect diffus, envahissant, échappant plus ou moins au domaine des choses contrôlables.[...] Les lettres qui en sont les supports graphiques, anarchiques comme celles de certains ballons, sont encore soulignées par une disposition typographique variable d'où toute recherche symétrique semble exclue» (Fresnault-Deruelle 1972a:34). Cette caractéristique intrinsèque du code pictural de la BD explique ainsi les emprunts à l'anglais, surtout dans une énumération de signes qui symbolisent une bataille par exemple et qui constituent à eux seuls tout le texte et le dessin de la vignette. Personne ne s'étonnera de ces agencements de graphèmes qui pourtant sont des plus étranges. D'où l'importance pour obtenir une bonne traduction dans un texte correct que le traducteur soit lui-même un lecteur de $\mathrm{BD}$ expérimenté. C'est à 
lui de choisir dans quel contexte linguistique et situationnel il doit utiliser l'une ou l'autre forme des doublets.

\subsection{Les interférences}

D’autre part, la connaissance insuffisante de la langue de départ, du français en l'occurrence, chez le traducteur entraîne des interférences dans la langue d'arrivée. Par exemple, nous pouvons citer:

- l'emploi de locutions traduites littéralement qui ne s'utilisent pas en espagnol (à moi! > ¡ a mi! pour demander de l’aide);

- ou des locutions empruntées au français: leur emploi en espagnol provoque un faux-sens, ce qui est le cas de l'interjection oh la la qui figure dans le texte espagnol $(A N: 42)$;

- ou encore l'exemple suivant:

attention! > ¡atención!, alors qu'on dirait: «¡cuidado!» ou « ¡ ojo!» dans cette situation, car ces interjections correspondent au registre familier du texte dans lequel apparaît «attention!» $(A N: 6)$.

La similitude entre les deux formes lexicales dans les deux langues facilite l'apparition des formes françaises dans le texte espagnol alors qu'en fait l'usage exigerait un autre terme en espagnol.

\subsection{La méconnaissance du sémantisme de l'interjection}

L'interjection fait partie du vocabulaire le plus difficile à assimiler et à mémoriser dans l'apprentissage d'une langue étrangère à cause de son caractère particularisant, qui la fait dépendre du locuteur qui l'utilise et de la situation dans laquelle il l'utilise. Très souvent, un apprenant retient le sens d'une interjection qu'il a découverte dans un texte ou une conversation mais oublie ce contexte. Le réemploi de cette interjection dans un autre contexte, en lui attribuant l'unique sens connu, peut évidemment mener à un contresens. Ainsi, dans notre corpus, il existe plusieurs traductions qui correspondent à l'une des acceptions de l'interjection française et qui malheureusement n'est pas celle qui convient dans la situation d'énonciation.

En effet, zut! (AN:18), qui dénote la contrariété qu'une personne ressent dans une situation ou face à une personne qui le dérange, ne peut pas être traduite par ¡buf!, qu'utiliserait une personne qui s'ennuie et qui soupire: on attendrait plutôt dans le corpus utilisé dans ces albums une interjection du type: «¡recorcho!»ou « $i$ mecachis!»

¡Chist! (ATG:44) ou ; glub! (AG:14) en aucun cas ne traduisent l'enthousiasme, la joie ni la satisfaction exprimées par l'interjection chic!, généralement accompagnée de gestes, de bonds et d'applaudissements.

De même, malheureux! (TT: 18) n'est pas une injure (qui signifierait bien dans ce cas «¡desgraciado!») mais, étant donné le contexte, plutôt une lamentation qui devrait être rendue par « ¡ infeliz!», qui exprime la malchance ou l'infortune d'un individu.

La traduction, enfin, de kai kai kai par guau guau ( $A G: 6$ et 41) montre que le traducteur ignore le sens de cette interjection qui dénote la peur, ou tout au moins qu'il l'a mal traduite: guau guau n'est que la représentation de l'aboiement du chien en général. Puisque cette interjection n'a pas d'équivalent en espagnol, elle aurait dû être conservée dans le texte espagnol, ou le traducteur aurait pu créer un son inarti- 
culé pour représenter l'image des dents qui claquent («KKKK» par exemple) ou une onomatopée pour exprimer le tremblement des lèvres («fffuuu»par exemple). La méconnaissance du sémantisme du mot français pousse inévitablement le traducteur à commettre des erreurs.

\subsection{Difficultés d'ordre stylistique}

Le dernier type de difficultés dues à la mise en parallèle de deux langues dans la BD est d'ordre stylistique.

Souvent, dans le genre satirique (les albums d'Astérix par exemple), l'auteur a recours à des jeux de mots qui agrémentent le texte pour provoquer le rire. Ceux-ci exigent généralement une reformulation totale du message pour conserver l'effet comique produit et la cohérence de la situation. Nul besoin de souligner l'importance de connaître le sens et les conditions d'emploi de l'expression. Faute de quoi, de procédé comique elle deviendrait désastre tragique dans la traduction.

Tel est le cas de poil au menton! qui rime avec «leçon» dans le texte et qui n'apporte absolument rien au message si ce n'est le comique du personnage qui veut avoir le dernier mot $(A G: 38)$ et qui est mal traduit par ¡ buena idea!. Le personnage qui répond à l'autre pour éviter que celui-ci n'ait le dernier mot, c'est-à-dire la répartie pour la répartie, obéit en somme à la volonté de l'auteur d'utiliser le langage pour s'évader de la réalité et se rapprocher du monde de la fantaisie où le «non-sens » est permis. Mais, en français, il existe une expression conventionnalisée (par le sens - ou le non-sens si l'on préfère —, et par la forme: il y a une rime), contrairement à l'espagnol. Le traducteur aurait dû oser proposer une réplique beaucoup plus absurde, réellement hors du contexte par le sens et du point de vue de la forme, facilement repérable par le lecteur.

Par contre, le ¡por ejemplo! (AN:9) produit effectivement le même effet que par exemple! puisque les deux expressions ont la même structure et la même signification que les expressions qui les précèdent dans le message:

$$
\begin{array}{lll}
\text { ¡por Júpiter! } & > & \text { par Jupiter!; } \\
\text { ipor Tutatis! } & > & \text { par Toutatis!; } \\
\text { ¡por ejemplo! } & >\text { par exemple!. }
\end{array}
$$

La locution «par exemple!» ramène les Dieux cités dans les expressions «Jupiter, Toutatis, etc. » au même niveau terre à terre qu'un mot commun et banal. Le comique a une base purement lexicale.

La difficulté dans les cas antérieurs consiste donc à respecter et à rendre de manière effective et efficace la valeur ludique que la $\mathrm{BD}$ confère à son langage.

\subsubsection{La situation d'énonciation}

Un autre facteur dont il faut tenir compte pour obtenir la meilleure des traductions possibles est la situation d'énonciation du message.

Il convient de souligner dans la BD l'importance des dialogues des personnages et l'importance du lecteur. En effet, les personnages parlent beaucoup mais leur discours n'a de sens que par rapport au lecteur; la langue de la BD étant celle de gens en situation, elle est forcément conventionnelle et suit une convention partagée par toute une communauté linguistique à laquelle appartient le lecteur. La BD est riche 
en stéréotypes qui se rencontrent aussi bien dans l'évocation de plantes, d'animaux, de phénomènes naturels, de personnages que dans le langage. Même les onomatopées qui nous paraissent les plus proches de la réalité sont, nous l'avons déjà évoqué, un fait culturel, c'est-à-dire conventionnel et stéréotypé.

L'interjection qui fait partie du discours suit donc elle aussi les règles et les lois de l'énonciation dans une langue déterminée. Et son sens, ce qu'elle dénote, se déduit de la situation du locuteur. Par conséquent, son emploi ne se circonscrit pas à quelques situations déterminées; au contraire, elle peut être utilisée dans un nombre considérable de situations dans lesquelles elle acquerra tour à tour un sens ou un autre. On ne peut pas considérer l'interjection comme un terme polysémique. De fait, elle n'a pas de valeur significative; c'est pourquoi la même forme interjective pourra offrir plusieurs sens, soit l'un soit l'autre, en fonction du contexte communicatif, linguistique ou non.

Tel est le cas en français du «ah!» ou du «aïe!». L'interjection ah! figure dixneuf fois dans Tintin et onze fois dans Astérix. Ah! est traduit par $;$ ah! neuf fois seulement. L'interjection aïe! apparaît quatre fois avec des valeurs différentes.

L'interjection ah! sert en français:

- à marquer un sentiment du locuteur qui veut attirer l'attention sur lui (ex. 1): ah! je vous trouve enfin $>$ ah, gracias a Dios que os encuentro (soulagement) (TSO: 43);

- à signaler que les propos qui viennent d'être dits le concernent (ex. 2): ah! bon, je comprends > claro, claro $(T 7 B: 13)$;

- à réprouver un dit de l'interlocuteur (ex. 3): ah vraiment? > ¿ah sí? (TSO: 6);

- à raviver la conversation (ex. 4): ah! oui... > pas de traduction $(T 7 B: 43)$.

En espagnol, l'interjection « $;$ ah ! s'emploie dans trois de ces situations (les trois premières) : elle peut exprimer toutes sortes de sentiments vifs comme la satisfaction, la joie, la douleur, l'admiration, la surprise, etc. (ex. 1); elle symbolise la compréhension et l'acceptation de la part du locuteur de ce qui vient d'être dit (ex. 2), et elle dénote la surprise, l'ironie ou la réticence quand elle est interrogative (ex. 3). Par contre, on utilise d'autres interjections telles que «bueno, pues» pour réorienter la conversation (ex. 4). Le traducteur aurait pu employer ces deux dernières interjections mais dans ce cas-là, il a préféré ne pas traduire le ah! français. La raison de ce choix repose sans doute sur les systèmes différents auxquels ont recours les deux codes linguistiques pour transcrire la langue orale dans la langue écrite: alors que le français multiplie les interjections et que, par conventionnalisme, on accepte l'idée que ce processus stylistique reproduit l'emploi à l'oral (cf. Ferrari [1983: 57] qui parle de «l'interjection rhétorique»), dans la langue écrite espagnole, on considère que les points d'exclamation suffisent à exprimer les nuances dues à l'intonation du locuteur sans avoir à ajouter les interjections.

L'interjection aïe! en français est la manifestation par excellence de la douleur. Seule ou dans une interjection composée de trois " aïe» ("aïe aïe aïe!»), elle indique aussi le pressentiment du locuteur qu’il va arriver quelque chose de néfaste ( «aïe aïe aïe! ça va chauffer!»), la crainte que suscite un risque immédiat («aïe! je ne comptais pas là-dessus!»). Dans les deux cas, l'interjection «ouille!» est synonyme de «aïe!»; il se produit seulement un changement du son vocalique. 
Dans les albums de Tintin, aïe (ou Haï), qui représente la douleur du capitaine devant une explosion (TT: 37) ou la peur de Tintin devant des patrouilles (aïe! les voilà!; aïe, une patrouille!, pages 33 et 38) est traduit dans les deux cas par ¡ Ay Dios mío! ou ¡Dios mío! seul).

Dans les aventures d'Astérix, l'auteur préfère l'emploi de ouille!, comme lorsque le druide se coupe le doigt $(A G: 16)$ traduit par ¡ huy!, ou à la page 42 , les cris de douleur dans les bagarres sont rendus par aïe aïe ou ouille ouille, traduits par ay ay ou uy uy. En effet, en espagnol, les deux interjections «ay» et «uy» manifestent, comme "aïe et ouille» en français, la peur et la douleur (de plus, «ay» symbolise par exemple le soulagement ou la joie; «uy» la satisfaction ou la stupeur).

Dans ces cas-là, le traducteur a donc bien vu et traduit les sentiments. Mais, dans d'autres cas, il a utilisé ces mêmes interjections espagnoles pour traduire des interjections françaises qui expriment d'autres sentiments, révélant ainsi qu'il confond le sens de certaines interjections de la langue de départ ou qu'il a mal analysé la situation. En effet, la situation, le dessin dans lequel apparaît l'interjection, est la clé pour déchiffrer la valeur de l'interjection.

Nous commenterons deux exemples:

- La vignette dans laquelle Astérix serre la main du druide qui dit: «houlà houlà houlà » $(A G: 8)$. Juste à la hauteur des mains sont dessinées des étoiles et des éclairs. L'image est ici l'expression de la douleur et on la retrouvera dans la BD traduite. Mais l'interjection du druide mêle à la sensation de la douleur le désir plus marqué d'interrompre le geste de l'interlocuteur: il s'agit d'une interjection orientée vers l'interlocuteur pour le faire agir ou plutôt dans ce cas pour qu'il cesse d'agir. La traduction serait donc, à notre avis: « ¡ ay! ¡ ya ya ya! ¡para, para!».

- La vignette dans laquelle Tintin tire sur la barbe du professeur Halambique pour le démasquer alors que celui-ci, surpris, crie: «ouh!» (TSO:22). Les étoiles dessinées tout autour transcrivent la douleur ressentie. Ici, le sens de l'interjection, plutôt que de manifester la douleur, est surtout d'interpeller l'autre en montrant son étonnement — provoqué, il est vrai, par la douleur. Elle équivaut donc à notre avis, à «oh! dites-donc!», qui devrait se traduire par « ¡ eh! ¡ oiga Ud!» (et non pas par « ¡ ay!» seulement).

Il est par conséquent très important d'analyser la situation de communication dans laquelle surgit l'énonciation car souvent, d'une part, les différentes interjections changent de valeur selon leur emploi et, d'autre part, le code pictural mène parfois à l'erreur de traduction parce que l'interjection manifeste un sentiment ajouté et non pas celui du dessin exclusivement.

À l'inverse, il arrive quelquefois que les différences existant entre les représentations graphiques d'une expression figée oblige le traducteur à ajouter une interjection. Par exemple, «voir les trente-six chandelles» est en français traduit graphiquement d'une manière littérale par des chandelles qui tournent autour de la tête du personnage. Mais, dans une BD espagnole, ou bien on transforme les chandelles en étoiles dans l'image ou bien on est forcé d'ajouter une interjection exprimant la douleur pour que le même sens soit conservé.

De plus, les différentes valeurs des interjections n'ont pas toujours d'équivalents dans l'autre langue, ce qui pose des difficultés au traducteur. Celui-ci décide alors de ne pas les traduire en espagnol parce qu'on ne les emploie pas aussi systématiquement qu'en français dans des situations données ou parce que leur emploi est consi- 
déré comme un processus stylistique conventionnel pour transcrire l'oral dans la langue française écrite (et cet emploi n'existe pas en espagnol).

\subsubsection{Le respect de la langue des personnages}

Le dernier facteur dont il faut tenir compte dans notre typologie des difficultés de traduction de l'interjection dans la $\mathrm{BD}$ est le fait qu'une $\mathrm{BD}$ est un récit, un genre littéraire qui fait vivre des personnages de fiction. Des personnes sont créées, qui possèdent un caractère propre, une personnalité propre et une façon de parler propre. La traduction de l'interjection qui est donc, nous le répétons une fois de plus, l'expression la plus vive de l'affectivité, des sentiments de l'individu, dépend de la connaissance des personnages de la part du traducteur. Celui-ci doit les analyser, les examiner afin de rendre dans la BD espagnole le même ensemble de caractéristiques qui, unies, constituent chacun d'entre eux. Le respect pour l'idiolecte de chaque personnage est primordial. Le choix d'un vocabulaire par opposition à un autre est inhérent au personnage.

Ainsi, par exemple, les jurons et les insultes du capitaine Haddock sont bien particuliers et cette particularité doit être conservée dans l'autre langue. Les mêmes processus de formation, c'est-à-dire la répétition des mêmes mots et la surcomposition des interjections constituées par l'accumulation de plusieurs locutions interjectives, les mêmes champs sémantiques (ici, ceux de la tempête, de la marine...) doivent être repris lorsque le capitaine parle en espagnol parce que ces interjections sont précisément la caractérisation de ce personnage et forment un leitmotiv dans son langage qui le rendent sympathique et cher au lecteur.

Un autre cas est celui de Tintin qui est le prototype du héros pour enfants, le héros débrouillard, intelligent, astucieux, hardi, courageux, poli, bien élevé, qui sert de modèle à une génération (ou plusieurs générations). Il est le seul à utiliser par exemple saperlipopette ou sapristi, euphémisme pour ne pas blasphémer. Ces interjections maintenant démodées (car trop puritaines) survivent cependant dans les contes actuels pour les enfants (cf. par exemple Les belles histoires de Bayard Presse adressées à des enfants de trois à sept ans), sans doute à cause de leurs sonorités amusantes qui plaisent aux enfants: trois répétitions de l'occlusive sur cinq syllabes prononcées dans «saperlipopette» ou deux fois le « $\mathrm{i}$ » dans «sapristi», un mot qui contient trois voyelles.

Le fait est que le traducteur devrait fournir un équivalent qui soit en mesure d'offrir les mêmes caractéristiques de l'original ou tout au moins d'en respecter le caractère ludique. La traduction par ¡ carambita! ou ¡ adiós! ou ¡ mecachis! est réussie pour saperlipopette!; ¡mecachis! ou ¡ canastos! l'est aussi pour sapristi. Par contre, les interjections ¡ vaya! ou ¡ hombre!, plus neutres, pourraient facilement être remplacées par les antérieures et respecteraient davantage la façon de parler du Tintin original.

Le transfert de la langue orale des personnages oblige donc le traducteur à réaliser un choix dans le corpus d'interjections qui peuvent prendre la même valeur en espagnol qu'en français dans une situation déterminée, ou à inventer des interjections impropres, c'est-à-dire à créer des néologismes de sens afin de cerner de plus près un personnage, de lui être le plus fidèle possible (nous avons déjà commenté ce besoin de créer en ce qui concerne les graphies de bruits et de sons inarticulés!). 


\section{Conclusion}

Les difficultés de traduction de l'interjection dans la BD peuvent donc être classées de la façon suivante:

- celles qui sont dues à la méconnaissance linguistique des deux systèmes mis en parallèle, et qui concernent par conséquent la morphologie des interjections, la sémantique et la stylistique;

- celles qui se dégagent de l'analyse de la situation d'énonciation (ou du manque d'analyse), étant donné que la valeur de l'interjection varie selon son emploi;

- celles qui proviennent du genre littéraire particulier dans lequel apparaît l'interjection, la bande dessinée et plus concrètement du type de héros présenté, héros qui inévitablement est très bavard et exprime à tout bout de champ ses émotions au moyen de l'interjection.

\section{CONCLUSION GÉNÉRALE}

Dans cet article, nous avons envisagé, d'une part, la complexité de l'interjection à cause de sa nature linguistique et, d'autre part, la difficulté de la traduire qui, logiquement, s'ensuit. En outre, l'inclusion des interjections de notre corpus dans un genre littéraire tel que la bande dessinée, qui possède un code pictural et verbal propre, augmente cette difficulté puisque le traducteur doit être en plus un lecteur expérimenté de bandes dessinées.

Les problèmes de traduction de l'interjection, que nous avons analysés et classés dans notre travail, découlent donc de deux caractéristiques de notre corpus : appartenir à la bande dessinée d'une part et à la langue orale d'autre part. Le premier type de problèmes s'ajoute à ceux que pose la méconnaissance des deux langues mises en parallèle et leurs cultures, de l'influence du contexte, de la situation d'énonciation, etc.

\section{NOTE}

1. Les albums du corpus et leurs abréviations respectives sont les suivants: Astérix le Gaulois $(A G)$, Le tour de Gaule d'Astérix (ATG), Astérix et les Normands (AN), Le Sceptre d'Ottokar (TSO), Les 7 boules de cristal (T7B) et Tintin au Tibet (TT).

\section{RÉFÉRENCES}

Alarcos Llorach, E. (1994): Gramática de la lengua española, Madrid, Espasa-Calpe.

Almela Pérez, R. (1990): Apuntes gramaticales sobre la interjección, Murcia, Publicaciones de la Universidad de Murcia.

Anscombre, J.C. (1985): «Onomatopées, délocutivité et autres blablas», Revue Romane, 20-2, p. $169-207$.

BARberis, J.M. (1992) : «Onomatopée, interjection: un défi pour la grammaire », L'information grammaticale, 53 , p. 52-57.

Beinhauer, W. (1991) : El español coloquial, Madrid, Gredos.

Benayoun, R. (1968): Le ballon dans la bande dessinée, Paris, Balland.

Benveniste, E. (1974): Problèmes de linguistique générale, Paris, Gallimard.

Cascon Martin, E. (1995): Español coloquial. Rasgos, formas y fraseología de la lengua diaria, Madrid, Edinumen.

Diccionario enciclopédico Larousse (1984): Barcelona, Planeta. 
Diccionario de la lengua española, Real Academia Española (1992): (20a ed.), Madrid, Espasa Calpe.

Ferrari, A. (1983) : «Interjecciones, exclamaciones y muletillas: el francés frente al español y sus modalidades regionales", Parallèles, 6, p. 55-61.

Fresnault-Deruelle, P. (1971): "Aux frontières de la langue: quelques réflexions sur les onomatopées de la bande dessinée», Cahiers de lexicologie, 18-1, p. 79-88.

- (1972a): La bande dessinée, Paris, Hachette.

- (1972b) : «La langue des bandes dessinées et leur contenu lexical», Le français dans le monde, 98, p. 14-19.

- (1972c): Dessins et bulles, Paris, Bordas.

Gubern, R. (1972): El lenguaje de los cómics, Barcelona, Península.

Hernandez Alonso, C. (1992): Gramática funcional del español, Madrid, Gredos.

Lamiquiz, V. (1975) : Lingüística española, Sevilla, Publicaciones de la Universidad de Sevilla.

Lope-Blanch, J.M. (1979): El concepto de oración en la lingüística española, México, Universidad Autónoma de México.

Lopez Garcia, A. (1989): Fundamentos de lingüistica perceptiva, Madrid, Gredos.

Lopez Garcia, A. et R. Morant (1995) : Gramática femenina, Madrid, Cátedra.

Martinez Alvarez, J. (1990): Las interjecciones, Logroño, Consejerería de Educación, Cultura y Deportes del Gobierno de la Rioja.

Olivier, C. (1994): «Les interjections et autres signes linguistiques comme marqueurs d'actes de prédication ", Champs du signe, 4, p. 215-231.

Renard, J.B. (1978): La bande dessinée, Paris, Seghers.

Robert, P. (1973): Le Petit Robert, Paris, Dictionnaires Le Robert.

Roca-Pons, J. (1980): Introducción a la gramática, Barcelona, Teide.

Santoyo, J.C. (1985): El delito de traducir, León, Universidad de León.

Sauvageot, A. (1964): Portrait du vocabulaire français, Paris, Larousse.

Tesniere, L. (1936): «Sur la classification des interjections», Mélanges, p. 343-352.

Toussaint, B. (1976): «Idéographie et bande dessinée», Communications, 24, p. 81-93.

Vigara Tauste, A.M. (1992): Morfosintáxis del español, Madrid, Gredos.

\section{Corpus}

Goscinny Et Uderzo (1961) : Astérix le Gaulois, Bruxelles, Dargaud.

- (1965): Le tour de Gaule d'Astérix, Bruxelles, Dargaud.

- (1967): Astérix et les Normands, Bruxelles, Dargaud.

— (1995): Asterix el Galo, Barcelona, Grijalbo-Dargaud.

- (1996): La vuelta a la Galia por Asterix, Barcelona,Grijalbo-Dargaud.

- (1997): Asterix y los Normandos, Barcelona, Grijalbo-Dargaud.

Hergé (1947): Le sceptre d'Ottokar, Barcelona, Ediciones del Prado.

- (1948): Les 7 boules de cristal, Barcelona, Ediciones del Prado.

- (1960): Tintin au Tibet, Barcelona, Ediciones del Prado.

- (1989): Las 7 bolas de cristal, Barcelona, Juventud.

— (1993): El cetro de Ottokar, Barcelona, Juventud.

- (1995): Tintín en el Tibet, Barcelona, Juventud. 